\title{
ALMOST TANGENT AND COTANGENT STRUCTURES IN THE LARGE
}

\author{
G. THOMPSON AND U. SCHWARDMANN
}

\begin{abstract}
We examine some global properties of integrable almost tangent and cotangent manifolds. In particular, we extend several results which essentially characterize tangent and cotangent bundles as, respectively, regular almost tangent and cotangent structures.
\end{abstract}

\section{INTRODUCTION}

In this paper we investigate some of the global features of almost tangent and almost cotangent manifolds. As the name suggests, these manifolds are defined by abstracting the key differential geometric structure of the tangent and cotangent bundle, respectively, of a differentiable manifold. They were introduced in 1960 and studied between then and the mid 1970 s by several authors mainly in Western Europe (see for example [3, 4, 5, 6, 10, 11, 12, 16]). However, an almost cotangent manifold which satisfies certain integrability conditions, an integrable almost cotangent structure, is synonymous with what is known in symplectic geometry as a polarization. Polarizations have been studied widely in their own right, without the intermediate notion of an almost cotangent structure (see $[14,15,24,25])$. This paper extends some previous results which measure to what extent integrable almost tangent and cotangent manifolds can actually deviate from tangent and cotangent bundles, respectively.

An outline of the paper is as follows. In $\S 2$ we study almost tangent and cotangent manifolds together, in terms of the theory of $G$-structures. Indeed we show (Lemma 2.1) that a manifold admits an almost tangent structure iff it admits an almost cotangent structure. We then consider some related $G$ structures and show that several familiar manifolds cannot carry almost tangent or cotangent structures.

$\S 3$ is concerned with integrable almost tangent structures. We review briefly a structure theorem (Theorem 3.3) and consider some extensions (Corollaries 3.4, 3.5 and Theorem 3.6). $\S 3$ concludes with an alternative characterization of the tangent bundle as an almost tangent structure in terms of the Liouville or dilation vector field.

Received by the editors August 26, 1987 and, in revised form, August 15, 1989.

1980 Mathematics Subject Classification (1985 Revision). Primary 53C15; Secondary 58F05. 
$\S 4$ consists of a parallel discussion for integrable almost cotangent structures. We also give two examples which illustrate the scope of the theorems and show finally that in dimensions 2 and 4 the universal cover of the total space of a regular almost cotangent manifold is covered by a cotangent bundle. With regard to notation, all manifolds are assumed to be paracompact and smooth, that is, of class $C^{\infty}$. Finally, we denote the interior product of a $p$-form $\alpha$ by a vector field $X$ by $i(X) \alpha$.

\section{Almost TANGENT AND ALMOST COTANGENT STRUCTURES AS $G$-STRUCTURES}

A smooth $2 m$-dimensional manifold $N$ is said to carry an almost tangent structure if it has a type $(1,1)$ tensor field $S$ with the property that at each point of $u$ in $N$, the kernel of $S_{u}$ (regarded as defining an endomorphism of the tangent space $T_{u} N$ ) coincides with its image. On the other hand, $N$ is said to carry an almost cotangent structure if it has a 2-form $\omega$ of maximal rank (an almost symplectic structure) and a Lagrangian distribution $\mathscr{L}$; that is to say, $\mathscr{L}$ is $m$-dimensional and $\omega$ vanishes when evaluated on a pair of vectors tangent to $\mathscr{L}$. As one would suspect from the name, the preeminent examples of almost tangent and cotangent structures are the tangent and cotangent bundles $T M$ and $T^{*} M$, respectively, of a smooth manifold $M$. These structures on $T M$ and $T^{*} M$ satisfy in addition certain integrability conditions, and we study such integrable almost tangent and cotangent structures in $\S \S 3$ and 4, respectively.

The almost tangent and almost cotangent structures on $N$ both determine and are determined by $G$-structures, that is to say, a reduction of the frame bundle $F(N)$ to a principal subbundle $B_{G}$ with structure group $G$ a closed subgroup of $\mathrm{GL}(2 m, \mathbb{R})$. (This is discussed in detail in $[3,5,8,23]$.) In the case of an almost tangent structure, $G$ consists of matrices of the form $\left[\begin{array}{ll}A & 0 \\ B & A\end{array}\right]$, with $A \in G L(m, \mathbb{R})$ and $B$ an arbitrary $m \times m$ matrix, as we briefly explain next. At each point $u$ in $N$, an almost tangent structure $S$ determines an $m$-dimensional subspace of $T_{u} N$, namely, $\operatorname{ker} S_{u}$. Choose a complement in $T_{u} N$ to $\operatorname{ker} S_{u}$ and a basis $\left(e_{i}\right)$ for it. Then $\left(e_{i}, S\left(e_{i}\right)\right)$ is a basis for $T_{u} N$, that is, a frame adapted to the almost tangent structure. One may easily verify that if $\left(e_{i}^{\prime}, S\left(e_{i}^{\prime}\right)\right)$ is another such frame, the two frames are related by a matrix of the form $\left[\begin{array}{ll}A & 0 \\ B & A\end{array}\right]$. Conversely, given a reduction of $F(N)$ to the group $G$, then as $G$ is the invariance group of the matrix $S=\left[\begin{array}{cc}0 & 0 \\ I_{m} & 0\end{array}\right]$ (where $I_{m}$ is the $m \times m$ identity matrix), $S$ may be defined as the type $(1,1)$ tensor which has at any point the matrix representation $S$ with respect to one of the frames determined by the $G$-structure and hence by the definition of $G$, with respect to any. Similarly, the existence of an almost cotangent structure on $N$ is equivalent to the reduction of $F(N)$ to a principal $G$-bundle, where $G$ is the group of matrices of the form $\left[\begin{array}{cc}A & 0 \\ B\left(A^{-1}\right)^{t}\end{array}\right]$ with $A \in G L(m, \mathbb{R})$ and $\left(A^{t} B\right)^{t}=A^{t} B$. (See $[6,23]$.)

We recall the following standard facts about $G$-structures (see $[19,22]$ ). 
Lemma 2.1. (i) An $n$-manifold $N$ is orientable iff the structure group of $F(N)$ is reducible to a subgroup of $G L^{+}(n, \mathbb{R})$ (matrices with positive determinant).

(ii) If the structure group of $F(N)$ is reducible to $H$, where $H$ is a closed subgroup of $G L(n, \mathbb{R})$, and $G$ is a closed subgroup of $G L(n, \mathbb{R})$, containing $H$, then the $H$-structure on $N$ corresponding to $H$ is uniquely extendible to a $G$-structure on $N$.

We see immediately from Lemma 2.1(i) that almost tangent and cotangent manifolds are orientable; for the determinants of matrices of the form $\left[\begin{array}{cc}A & 0 \\ B & A\end{array}\right]$ and $\left[\begin{array}{cc}A & 0 \\ B\left(A^{-1}\right)^{t}\end{array}\right]$ are respectively $(\operatorname{det} A)^{2}$ and 1 and in particular are both positive. Using part (ii) of Lemma 2.1 we also obtain the following result.

Theorem 2.2. $A$ 2m-manifold $N$ admits an almost tangent structure iff it admits an almost cotangent structure.

Proof. Suppose $N$ has an almost tangent structure with corresponding $(1,1)$ tensor $S$. If we choose a Riemannian metric on $N$, we may consider at each point $u$ of $N$ the unique orthogonal complement to $\operatorname{ker} S$. This results in a reduction of the structure group to a subgroup $H$ of $S O(2 m)$ of the form $\left[\begin{array}{ll}A & 0 \\ 0 & A\end{array}\right]$. Evidently $A \in O(m)$ and $H$ is isomorphic to $O(m) \times O(m)$.

Similarly, starting from an almost cotangent structure by choosing a Riemannian metric we can reduce the structure group to a matrix subgroup of $S O(2 \mathrm{~m})$ of the form $\left[\begin{array}{cc}A & 0 \\ 0 & \left(A^{-1}\right)^{t}\end{array}\right]$. It follows that $A \in O(m)$, and thus this latter subgroup coincides with $H$ defined above.

We can make explicit the correspondence between the tensorial objects, implied by Theorem 2.2, as follows. Given an almost tangent structure $S$ and a Riemannian metric $g$ on $N$ define the endomorphism field $T$ as follows, where $(\operatorname{ker}(S))^{\perp}$ denotes the distribution consisting of vectors orthogonal to the distribution $\operatorname{ker}(S)$ :

$$
\begin{aligned}
& J X=S X \quad\left(X \in(\operatorname{ker}(S))^{\perp}\right), \\
& J X=-\left(S^{-1} X\right) \cap(\operatorname{ker} S)^{\perp} \quad(X \in \operatorname{ker}(S)) .
\end{aligned}
$$

Then $\omega$ defined by

$$
\omega(X, Y)=g(X, J Y)-g(Y, J X)
$$

is seen to be an almost symplectic 2-form with $\operatorname{ker} S$ as Lagrangian distribution. Conversely, it is easy to see that starting from an almost symplectic 2-form $\omega$ with Lagrangian distribution $\mathscr{L}$ we may define an almost tangent structure $S$ with kernel $\mathscr{L}$.

Notice that the endomorphism field $J$ is an almost complex structure, that is, $J^{2}=-I$ (the identity tensor on $N$ ). Furthermore, $N$ carries an almost Hermitian structure $h$, which is to say a Riemannian metric such that for all $X, Y \in T N$,

$$
h(J X, J Y)=h(X, Y)
$$


In fact, $h$ may be defined by

$$
h(X, Y)=g(X, Y)+g(J X, J Y) .
$$

The reduction of the frame bundle $F(N)$ of the $2 m$-dimensional manifold $N$ to a $\left[\begin{array}{cc}A & 0 \\ 0 & A\end{array}\right]$-bundle, with $A \in O(m)$, is equivalent to $T N$ splitting as a Whitney sum of two isomorphic subbundles, a condition which is quite stringent. For example, we can assert that no even-dimensional sphere $S^{2 m}$ can support an almost tangent structure (since $T S^{2 m}$ admits no proper subbundles [2, 21]) nor can $\mathbb{R} P^{2 m}$ (in fact we already know this because $\mathbb{R} P^{2 m}$ is nonorientable).

It is also natural to ask whether $\mathbb{C} P^{m}$ can support an almost tangent structure. This certainly cannot be the case for $m$ odd, because since $\mathbb{C} P^{m}$ is orientable, the splitting of $T \mathbb{C} P^{m}$ as a Whitney sum of two odd-dimensional subbundles would entail the vanishing of the Euler class, which is false $[2$, p. 126;21, p. 280]. On the other hand, suppose that $m$ is even and that $T \mathbb{C} P^{m}$ splits as a Whitney sum $\xi \oplus \xi$ for some real rank $m$ vector bundle $\xi$ over $M$. Let $w_{i}$ denote the $i$ th Stiefel-Whitney cohomology class of $\xi$. Then the total StiefelWhitney class of $\xi \oplus \xi$ is easily seen to be $1+w_{1}^{2}+w_{2}^{2}+\cdots+w_{m}^{2}$. However, the total Stiefel-Whitney class of $T \mathbb{C} P^{m}$ is the $\bmod 2$ reduction of the total Chern class. Now the latter is well known to be $(1+x)^{m+1}$, where $x$ is the first Chern class and of course $x^{m+1}$ but no smaller power of $x$ is zero [2, 21]. Since $m$ is even, on equating the total Stiefel-Whitney classes of $\xi \oplus \xi$ and $T \mathbb{C} P^{m}$, we find that

$$
x \equiv w_{1}^{2} \bmod 2 \text {. }
$$

However, since $\mathbb{C} P^{m}$ is simply connected, any vector bundle over it must be orientable and hence $w_{1}(\xi)$ must be zero. This contradicts $(2.5)$, and we reach the conclusion that $T \mathbb{C} P^{m}$ cannot split as a Whitney sum $\xi \oplus \xi$.

\section{INTEGRABLE ALMOST TANGENT STRUCTURES IN THE LARGE}

In this section we review some well-known global features of integrable almost tangent structures and present several new results. It is convenient at this stage to consider tangent and cotangent structures separately, and $\S 4$ is devoted to the latter.

A $G$-structure $B_{G}$ on a manifold $N$ is said to be flat or integrable if, about every point $u$ of $N$, there exists a coordinate system $\left(x^{i}\right)$ such that the corresponding moving frame $\left(\partial / \partial x^{i}\right)$ defines a local section of $B_{G}$ over $N$. In the case of an almost tangent structure, it is known that integrability is equivalent to the vanishing of the Nijenhuis tensor of the associated tensor fields [3, 8, 11]. If the almost tangent structure $S$ is integrable, then so is the distribution $\operatorname{ker}(S)=\operatorname{im}(S)$ (in the sense of Frobenius' theorem). We call an integrable almost tangent structure $(N, S)$ for which $N$ is the total space of a smooth fibre bundle with base $M$, submersion $\pi: N \rightarrow M$ and whose fibres coincide with the leaves of $S$, regular. We shall say that $(N, \pi, M, S)$ is a regular almost tangent manifold. 
We consider next some of the key geometric properties of the tangent bundle $T M$ and show in particular that it is a regular almost tangent manifold. First, $T M$ (as well as $T^{*} M$ ), by virtue of being a vector bundle, possesses a canonical vertical vector field $\Delta_{0}$ known as the Liouville field [12, 16, 18]. The group of nonzero real numbers acts on the fibres of $T M$ by way of scalar multiplication, and $\Delta_{0}$ is just the infinitesimal generator corresponding to this action.

Next, let $X$ be a vector field on $M$. If $\phi_{t}$ is its local flow on $M$, then $T\left(\phi_{t}\right)$ is the local flow of a vector field $X^{c}$ on $T M$, known as the complete lift of $X$. There is also a construction known as the vertical lift of vector fields. We denote the canonical submersion map from $T M$ to $M$ by $\pi$ and suppose we are given a point $x \in M$ and an element $X \in T_{x} M$. Then note that $T_{u}\left(\pi^{-1}(x)\right.$ ) (the tangent space to the fibre at $u \in T M)$ is canonically isomorphic with the vector space $\pi^{-1}(x)$, which is just $T_{x} M$. Thus to each $X$ in $T_{x} M$ there corresponds a unique vertical tangent vector denoted by $X^{v}$ at $u$ and called the vertical lift of $X$. Repeating this construction at each point $u$ in $\pi^{-1}(x)$ gives a vector field $X^{v}$ on $\pi^{-1}(x)$ and if $X$ is a vector field on $M$, we obtain a vertical vector field $X^{v}$ on the whole of $T M$. (The various interpretations of the symbol $X^{v}$ causes little difficulty in practice.)

The vertical lift construction enables us to define easily the almost tangent structure $S_{0}$ on $T M$. In fact, if $u \in T M$, then $S_{0}$ at $u$, as an endomorphism of $T_{u} T M$, is simply the composition of the linear map $\pi_{*}: T_{u} T M \rightarrow$ $T_{x} M(\pi(u)=x)$ with the vertical lift map from $T_{x} M$ to $T_{u} T M$. It is not difficult to see that a diffeomorphism $T \phi$ of $T M$ induced from a diffeomorphism $\phi$ of $M$ preserves $S_{0}$, as does a diffeomorphism of $T M$ which consists of a translation in each fibre [8]. Conversely, a diffeomorphism of $T M$ which preserves $S_{0}$ can be written uniquely as the composition of a diffeomorphism $T \phi$, for some diffeomorphism $\phi$ of $M$, followed by a diffeomorphism of $T M$ consisting of translations in each fibre.

Suppose now that $(N, \pi, M, S)$ is a regular almost tangent manifold. Then we may use $\mathscr{S}$ to define a vertical lift construction. To be specific, if $u \in N$ with $\pi(u)=x$ and $X \in T_{x} M, X^{v}$ is the unique element of $T_{u} N$ defined by

$$
X^{v}=S_{u}(\widehat{X})
$$

where $\widehat{X}$ is an element of $T_{u} N$ which projects via $\pi_{*}$ onto $u$; since the kernel of $\pi_{*}$ coincides with $\operatorname{ker} S$, the choice of such $X$ is immaterial. We collect together several facts about vertical lifts which are proved in [8], $X$ and $Y$ being arbitrary vector fields on $M$.

Lemma 3.1. (i) Vertical lifts are indeed vertical, that is, $\pi_{*} X^{v}=0$. Moreover, the vertical lift construction establishes, for each point $u \in N$ such that $\pi(u)=x$, an isomorphism between $T_{x} M$ and $T_{u}\left(\pi^{-1}(x)\right.$ ) (the tangent space to the fibre at $u$ );
(ii) $\left[X^{v}, Y^{v}\right]=0$;
(iii) $L_{X^{v}} S=0$;
(iv) On TM itself $L_{X^{c}} S_{0}=0$. 
We shall also need the following elementary lemma from Riemannian geometry.

Lemma 3.2. Let $(M, g)$ be a Riemannian manifold and $K$ be a Killing vector field whose length is constant. Then $K$ is a geodesic field.

Proof. Let $\nabla$ be the Levi-Civita connection of $g$. The fact that $K$ is, respectively, Killing and of constant length implies that

$$
\begin{gathered}
g\left(\nabla_{X} K, Y\right)+g\left(X, \nabla_{Y} K\right)=0, \\
g\left(\nabla_{X} K, K\right)=0,
\end{gathered}
$$

for arbitrary vector fields $X$ and $Y$ on $M$. Taking $Y=K$ in (3.2) gives

$$
g\left(X, \nabla_{K} K\right)=0 \text {, }
$$

and the result follows since $X$ is arbitrary.

We now present a structure theorem for regular almost tangent manifolds. This theorem is essentially the same as the main results in $[3,8]$ and hence we are content simply to outline the proof.

Theorem 3.3. Let $(N, \pi, M, S)$ be a regular almost tangent manifold. Then the model fibre of the bundle is a generalized cylinder, that is, of the form $T^{\rho} \times \mathbb{R}^{m-\rho}$ ( $T$ being a $\rho$-torus with $0 \leq \rho \leq m$ ). Suppose further that the fibres of $\pi$ are complete (as locally affine spaces) and simply connected. Then $N$ is naturally diffeomorphic to the affine tangent bundle of $M$; that is, $N$ is an affine bundle modeled on the vector bundle TM.

(Sketch of proof.) By Lemma 3.1(i), a choice of positive definite scalar product on $T_{x} M$ induces a Riemannian metric, denoted by $g_{x}$, on $\pi^{-1}(x)$. Also, if $X, Y \in T_{x} M$ it is evident that $g_{x}\left(X^{v}, Y^{v}\right)$ is constant on $\pi^{-1}(x)$ and, in particular, that $X^{v}$ has constant length on $\pi^{-1}(x)$. Furthermore, every such $X^{v}$ on $\pi^{-1}(x)$ is a Killing vector field of $g_{x}$. For let $Y^{v}$ and $Z^{v}$ be two other vertical lifts. Then

$$
L_{X^{v}}\left(g_{x}\left(Y^{v}, Z^{v}\right)\right)=\left(L_{X^{v}} g_{x}\right)\left(Y^{v}, Z^{v}\right)
$$

by Lemma 3.1(ii) and the fact that the Lie derivative operator $L$ acts as a derivation. However, $g_{x}\left(Y^{v}, Z^{v}\right)$ is constant as we explained above, and so

$$
L_{X^{v}} g_{x}=0
$$

that is, $X^{v}$ is a Killing field of $g_{x}$.

It follows now by Lemma 3.2 that each vertical lift $X^{v}$ is geodesic for $g_{x}$. Thus a basis in $T_{x} M$ determines a complete parallelism on $\pi^{-1}(x)$, since vertical lifts commute. The Riemannian manifold $\pi^{-1}(x)$ is therefore flat and, if geodesically complete, diffeomorphic to a quotient space of $\mathbb{R}^{m}$ by a discrete 
subgroup acting properly discontinuously. Since we are assuming that $\pi^{-1}(x)$ is simply connected, however, $\pi^{-1}(x)$ must be diffeomorphic to $\mathbb{R}^{m}$.

It is now not difficult to see that $\pi^{-1}(x)$ may be identified with $T_{x} M$. Indeed, as a consequence of what has been established so far, it follows that the geodesic exponential map based at any point $u$ in $\pi^{-1}(x)$ is a diffeomorphism. Applying this correspondence on each fibre of $\pi$ shows that $N$ is an affine space bundle modeled on $T M$.

Our immediate objective now is to obtain some generalizations of Theorem 3.3. Before we can do that, however, we must consider a class of regular almost tangent manifolds whose almost tangent structures are derived from the corresponding structures on tangent manifolds $T M$. Suppose that $M$ admits $\rho$ vector fields $X_{1}, X_{2}, \ldots, X_{\rho}$, which are everywhere linearly independent over the ring of smooth functions on $M$. By choosing a Riemannian metric $g$ on $M$, we may write $T M$ as the Whitney sum $\xi \oplus \xi^{\perp}$, where $\xi$ is the trivial rank $\rho$ subbundle of $T M$ determined by the $X_{i}$ 's and $\xi^{\perp}$ is its orthogonal complement. For each $x \in M, X_{1}(x), X_{2}(x), \ldots, X_{\rho}(x)$ can be used to generate a $\rho$-dimensional lattice in $T_{x} M$ and hence there is a discrete subgroup $\Gamma$ of $G L(m, \mathbb{R})$ isomorphic to $\mathbb{Z}^{\rho}$ which acts fibrewise on $T M$ by translation. We denote the resulting quotient space $T M / \Gamma$ and it is clear that $T M$ is a covering space of $T M / \Gamma$. Furthermore, since the group $\Gamma$ consists of fibrewise translations, $S_{0}$ passes to the quotient to define an almost tangent structure $S$ on $T M / \Gamma$. Indeed $T M / \Gamma$ has the structure of a fibre bundle over $M$ with model fibre diffeomorphic to the generalized cylinder $T^{\rho} \times \mathbb{R}^{m-\rho}$ and $T M / \Gamma$ with $S$ satisfies all the requisite properties of a regular almost tangent structure.

By reexamining the proof of Theorem 3.3, we can derive the following corollaries.

Corollary 3.4. Let $(N, \pi, M, S)$ be a regular almost tangent structure with model fibre $T^{\rho} \times \mathbb{R}^{m-\rho}$. Suppose further that $N$ admits a smooth section $s$ over $M$. Then $T M$ is a covering space of $N$. Indeed $(N, \pi, M, S)$ is equivalent as an almost tangent manifold to $T M / \Gamma$, where $\Gamma$ is a group isomorphic to $\mathbb{Z}$ in the manner described above.

Corollary 3.5. Let $(N, \pi, M, S)$ be a regular almost tangent structure.

(i) If the model fibre is compact and a smooth section of $N$ over $M$ exists, then $M$ is parallelizable.

(ii) If $N$ is compact and a smooth section of $N$ over $M$ exists, then $M$ is parallelizable.

Proof. Part (ii) is a special case of (i), since if $N$ is compact, each fibre, being a closed subset of $N$, is also compact. To prove part (i) we note that by Corollary 3.4 the fibre must be $T^{m}$ and $\Gamma$ must be $\mathbb{Z}^{n}$. But then by definition, $T M / \Gamma$ is constructed by quotienting in each fibre of $T M$ by a lattice determined by $m$ linearly independent vector fields on $M$. 
Suppose again that $(N, \pi, M, S)$ is a regular almost tangent structure but that we do not assume the existence of a section of $N$ over $M$. Consider the universal cover $\widetilde{M}$ of $M$ and the corresponding covering map $\tau: \widetilde{M} \rightarrow$ $M$. Then it is not difficult to see that the induced bundle $\tau^{-1}(N)$, which is a covering space of $N$, is a regular almost tangent manifold with base $M$. Again, we may consider the covering space $\widetilde{N}$ of $\tau^{-1}(N)$ and it is easy to see that $\widetilde{N}$ is the total space of a regular almost tangent manifold with base $\widetilde{M}$ and we denote the corresponding $1-1$ tensor by $\widetilde{S}$ and bundle submersion by $\tilde{\pi}$. Moreover, the model fibre of $\tau^{-1}(N)$ over $M$ is diffeomorphic with the model fibre of $N$ over $M$, and the model fibre of $\widetilde{N}$ over $\widetilde{M}$ must be a covering space of the model fibre of $\tau^{-1}(N)$ over $\widetilde{M}$. These remarks show that, at least in a preliminary classification of regular almost tangent manifolds, one may as well assume that the total space and base space are simply connected; the others are obtained from these as quotients.

We now turn to the regular almost tangent manifold $(N, \pi, M, S)$ and denote the model fibre by $F$ and consider the fibre homotopy sequence (see, for example, [2])

$$
\cdots \pi_{k}(F) \rightarrow \pi_{k}(N) \rightarrow \pi_{k}(M) \rightarrow \pi_{k-1}(F) \rightarrow \pi \cdots \pi_{0}(N) \rightarrow \pi_{0}(M) .
$$

Now since $F$ is of the form $T^{\rho} \times \mathbb{R}^{m-\rho},(3.7)$ implies that $\pi_{k}(N)$ is isomorphic to $\pi_{k}(M)$ for $k \geq 3$ and also, if $\pi_{1}(N)=0$, that $\pi_{1}(F)$ is isomorphic to $\pi_{2}(M) / \pi_{2}(N)$. By applying Theorem 3.3 to $(N, \pi, M, S)$, we obtain the following theorem.

Theorem 3.6. Let $(N, \pi, M, S)$ be a regular integrable almost tangent structure with fibre $F$. Then $\pi_{k}(N)$ and $\pi_{k}(M)$ are isomorphic for $k \geq 3$ and $\pi_{2}(N)$ is a subgroup of $\pi_{2}(M)$. Moreover, if $\pi_{1}(N)$ is trivial, $\pi_{1}(F)$ is isomorphic with $\pi_{2}(M) / \pi_{2}(N)$. In that case, if either $\pi_{2}(M)$ is trivial or $\pi_{2}(N)$ and $\pi_{2}(M)$ are isomorphic, then $(N, \pi, M, S)$ is equivalent as an almost tangent manifold to $T M$.

We shall give an alternative characterization of the almost tangent structure on $T M$ in terms of the Liouville field $\Delta_{0}$ introduced towards the beginning of this section. This result strengthens a result of Brickell and Clark [3, Theorem 3]. We shall need two lemmas and the following identity relating $S_{0}$ and $\Delta_{0}$ :

$$
L_{\Delta_{0}} S_{0}=-S_{0} \text {. }
$$

Lemma 3.7. Let $(N, \pi, M, S)$ be a regular almost tangent structure. Suppose that $\Delta$ is a vector field on $N$ such that $L_{\Delta} S=-S$. Then there exists a vertical vector field $\bar{\Delta}$ on $N$ which satisfies $L_{\bar{\Delta}} S=-S$.

Proof. Choose a good cover [2] $\left\{U_{\alpha}\right\}$ of $M$ and a partition of unity $\left\{\lambda_{\alpha}\right\}$ subordinate to $\left\{U_{\alpha}\right\}$. Then since $\left\{U_{\alpha}\right\}$ is a good cover, each $U_{\alpha}$ is diffeomorphic to $\mathbb{R}^{m}$ and hence $\pi^{-1}\left(U_{\alpha}\right)$ admits a smooth section over $U_{\alpha}$. Now $\pi^{-1}\left(U_{\alpha}\right)$ is a regular almost tangent structure and so by Corollary $3.4, T U_{\alpha}$ is a covering space of $\pi^{-1}\left(U_{\alpha}\right)$. 
Now consider the vector field $\Delta$ on $N$ and let $V$ be an arbitrary vertical field on $N$ so that

$$
S V=0 .
$$

Differentiating (3.9) along $\Delta$ and using the fact that $L_{\Delta} S=-S$ gives

$$
S[\Delta, V]=0 .
$$

Thus $[\Delta, V]$ is vertical and since $V$ was an arbitrary vertical field, $\Delta$ must be projectable to, say, $X$ on $M$.

Let $X_{\alpha}$ denote the complete lift of $X_{\alpha}$ to $T U_{\alpha}$ and let the projection from $T U_{\alpha}$ to $\pi^{-1}\left(U_{\alpha}\right)$ be denoted by $\rho$. Then $\pi_{*}\left(\rho_{*} X_{\alpha}-\Delta\right)=0$ and so, in particular, $X_{\alpha}$ is $\rho$-projectable to $\pi^{-1}\left(U_{\alpha}\right)$. Now since $X_{\alpha}$ is a complete lift on $T U_{\alpha}$, the Lie derivative along $\rho_{*} X_{\alpha}^{c}$ of $S$ is zero and moreover $\rho_{*} X_{\alpha}^{c}$ is $\pi$-related to $X$. Thus setting $\Delta_{\alpha}=\Delta-\rho_{*} X_{\alpha}$ gives

$$
\begin{aligned}
L_{\Delta_{a}} S & =L_{\Delta} S-L_{\rho_{*} X_{\alpha}^{c}} S \\
& =-S-0 \\
& =-S .
\end{aligned}
$$

Thus $\Delta_{\alpha}$ is the required vertical vector field on $U_{\alpha}$. To obtain such a vector field defined on all of $M$, define $\bar{\Delta}$ to be $\sum_{\alpha} \lambda_{\alpha} \Delta_{\alpha}$, where $\left\{\lambda_{\alpha}\right\}$ is the partition of unity subordinate to $\left\{U_{\alpha}\right\}$. Then the following identity holds:

$$
L_{\bar{\Delta}} S=\sum_{\alpha}\left(\lambda_{\alpha} L_{\Delta_{\alpha}} S-\Delta_{\alpha} \otimes S\left(d \lambda_{\alpha}\right)+S\left(\Delta_{\alpha}\right) \otimes d \lambda_{\alpha}\right) .
$$

From (3.11) we easily conclude that $L_{\bar{\Delta}} S=-S$ because $S\left(d \lambda_{\alpha}\right)=0$ and $S\left(\Delta_{\alpha}\right)=0$, since $d \lambda_{\alpha}$ are basic 1-forms on $N$ and $\Delta_{\alpha}$ are vertical, respectively.

Lemma 3.8. Let $(M, g)$ be a complete Riemannian manifold. Suppose that $(M, g)$ possesses a homothetic vector field (or indeed simply a finite homothetic motion). Then $(M, g)$ is isometric to Euclidean space with its standard inner product.

For the proof of Lemma 3.8 we refer to [1, 20].

Theorem 3.9. The regular almost tangent structure $(N, \pi, M, S)$ is equivalent to the almost tangent structure on TM iff there exists a vector field $\Delta$ on $N$ such that $L_{\Delta} S=-S$.

Proof. The necessity is clear from (3.8). Suppose conversely that $\Delta$ on $N$ satisfies $L_{\Delta} S=-S$. Then by Lemma 3.7, without loss of generality, $\Delta$ may be assumed to be vertical. Now take any point $x \in M$, a positive definite scalar product on $T_{x} M$, and denote the resulting Riemannian metric on $\pi^{-1}(x)$ by $g_{x}$ (compare the proof of Theorem 3.3).

Next, starting from the definition $S \widehat{X}=X^{v}$, Lie-differentiating along $\Delta$ and using the fact that $L_{\Delta} S=-S$, gives

$$
\left[\Delta, X^{v}\right]=-X^{v}+S[\Delta, X] .
$$


But $X$ is projectable and so $[\Delta, X]$ is vertical; so (3.12) reduces to

$$
\left[\Delta, X^{v}\right]=-X^{v} \text {. }
$$

Finally, consider the Lie derivative of $g_{x}\left(X^{v}, Y^{v}\right)$ along $\Delta$ on the fibre $\pi^{-1}(x)$. We obtain

$$
\begin{gathered}
\Delta\left(g_{x}\left(X^{v}, Y^{v}\right)\right)=0, \\
\Rightarrow\left(L_{\Delta} g_{x}\right)\left(X^{v}, Y^{v}\right)+g_{x}\left(\left[\Delta, X^{v}\right], Y^{v}\right)+g_{x}\left(X^{v},\left[\Delta, Y^{v}\right]\right)=0 .
\end{gathered}
$$

Now from (3.13) we obtain

$$
\left(L_{\Delta} g_{x}\right)\left(X^{v}, Y^{v}\right)=2 g_{x}\left(X^{v}, Y^{v}\right),
$$

or since $X, Y$ are arbitrary in $T_{x} M$,

$$
L_{\Delta} g_{x}=2 g_{x} \text {. }
$$

Equation (3.17) says that $\Delta$ acts as a homothetic vector field on the complete Riemannian manifold $\left(\pi^{-1}(x), g_{x}\right)$. The theorem now follows from Lemma 3.8 and Theorem 3.3.

We conclude this section by noting that Theorem 3.9 answers a question suggested by a recent paper of Crampin and Ibort [9]. They point out that any regular almost tangent structure has associated to it a natural cohomology defined on the space of vector-valued forms which preserve the subspace of basic forms and are "of type $i_{*}$ " (see [9]). The almost tangent structure $S$ itself defines a cohomology class, which in the case of the tangent bundle is the zero class. Theorem 3.9 in effect provides the converse of this result; namely, if a regular almost tangent structure $(N, \pi, M, S)$ is such that $S$ defines a trivial element in cohomology, then $(N, \pi, M, S)$ is equivalent to $\left(T M, \pi_{0}, M, S_{0}\right)$. The condition for $S$ to define a trivial cohomology class is precisely that there should exist vector field $\Delta$ such that $L_{\Delta} S=-S$.

\section{INTEGRABLE ALMOST COTANGENT STRUCTURES IN THE LARGE}

We proceed by considering the global properties of almost cotangent structures. We emphasized in $\S 2$ the similarity of almost tangent and almost cotangent structures and the results of this section run as parallel as is possible to those of $\S 3$. However, that very similarity will enable us to be much briefer in this section.

Given an almost cotangent structure $(\omega, \mathscr{L})$ on a $2 m$-manifold $N$, it is well known that the associated $G$-structure on $N$ is integrable iff $\omega$ is integrable in the sense that $d \omega$ is zero and $\mathscr{L}$ is an involutive distribution $[6,13]$. In that case we shall say that $(\omega, \mathscr{L})$ is regular if $N$ is the total space of a smooth fibre bundle with base $M$, submersion $\Pi: N \rightarrow M$, whose fibres coincide with the leaves of $\mathscr{L}$. We shall say that $(N, \Pi, M, \omega)$ is a regular almost cotangent manifold. We emphasize that the weaker notion of a symplectic manifold with 
a foliation whose leaves are Lagrangian submanifolds, is known in the literature as a polarization $[14,24]$.

Again the preeminent example of a regular almost cotangent manifold is the cotangent bundle $T^{*} M$ of a smooth manifold $M$. We briefly review some of the geometry of $T^{*} M$. We already noted in $\S 3$ that $T^{*} M$ has a Liouville field $\Delta_{0}$, by virtue of being a vector bundle. We denote the canonical submersion from $T^{*} M$ to $M$ by $\Pi$. The canonical 1 -form $\theta_{0}$ on $T^{*} M$ is characterized by the condition that for all $X \in T_{p} T^{*} M$,

$$
\left\langle X, \theta_{0}\right\rangle_{p}=\left\langle\Pi_{*} X, p\right\rangle_{x}
$$

where $x \in M, p \in T_{x}^{*} M$. The canonical 2-form $\omega_{0}$ is simply $d \theta_{0}$. Now suppose that $x \in M$ and that $\alpha \in T_{x}^{*} M$. Then for $p \in \Pi^{-1}(x)$ there is a unique element $\alpha^{v}$ of $T_{p} T^{*} M$ which satisfies

$$
i\left(\alpha^{v}\right) \omega_{0}=\Pi^{*} \alpha,
$$

where $\Pi^{*}: T_{x}^{*} M \rightarrow T_{p}^{*} T^{*} M$. We call $\alpha^{v}$ the vertical lift of $\alpha$, and we also obtain a vector field $\alpha^{v}$ on $\Pi^{-1}(x)$ and a vector field $\alpha^{v}$ on $T^{*} M$ if $\alpha$ is a 1form on $M$. It follows easily from the fact that the fibres of $\Pi$ are Lagrangian submanifolds of $\omega_{0}$ that $\alpha^{v}$ (in any of its interpretations) is indeed vertical and that the Lie bracket of two such vertical lift fields is zero.

Next, suppose that $(N, \Pi, M, \omega)$ is a regular almost cotangent structure. Then there is also a well defined vertical lift construction whereby a covector (or 1-form) on $M$, induces a vertical vector (or vector field) $\alpha^{v}$, the vertical lift of $\alpha$, on $N$ by the condition

$$
i\left(\alpha^{v}\right) \omega=\Pi^{*} \alpha .
$$

Again, the Lie bracket $\left[\alpha^{v}, \beta^{v}\right]$ of two such vertical lift fields is zero, just as in the case of a cotangent bundle. (For further details on the vertical lift see [15, 23].)

The following theorem is the cotangent analogue of Theorem 3.3. (For more details see $[15,23,25]$.)

Theorem 4.1. Let $(N, \Pi, M, \omega)$ be a regular integrable almost cotangent structure. Then the model fibre of the bundle is a generalized cylinder, that is, of the form $T^{\rho} \times \mathbb{R}^{m-\rho}(0 \leq \rho \leq m)$. Suppose further that the fibres of $\Pi$ are complete (as locally affine spaces) and simply connected. Then $N$ is naturally diffeomorphic to the affine cotangent bundle of $M$. Furthermore, there exists a symplectic diffeomorphism $\Phi: N \rightarrow T^{*} M$ such that $\omega=\Phi^{*} \omega_{0}+\Pi^{*} \phi$, where $\phi$ is closed and the class $[\phi]$ in $H^{2}(M, \mathbb{R})$ characterizes $(N, \Pi, M, \omega)$ up to equivalence. In particular, $(N, \Pi, M, \omega)$ is equivalent to $\left(T^{*} M, \Pi_{0}, M, \omega_{0}\right)$ iff $[\phi]=0$.

We wish next to consider the almost cotangent analogue of the quotient almost tangent structures $T M / \Gamma$ presented in $\S 3$. We begin by reminding the 
reader first, however, of the well-known results which states that a diffeomorphism of $T^{*} M$ consisting of translation in the fibres of $\Pi$ by a closed 1 -form $\alpha$ on $M$, that is, the map $p \mapsto p+\alpha$, is a symplectomorphism, though it of course does not preserve $\theta_{0}$.

Suppose next that $M$ admits $\rho$ closed 1-forms which are everywhere linearly independent (over the ring of smooth functions on $M$ ). Then as in the case of the almost tangent structure on $T M$, there exists a discrete subgroup $\Gamma$ of $G L(m, \mathbb{R})$ isomorphic to $\mathbb{Z}^{\rho}$ which acts fibrewise by translation on $T^{*} M$. The symplectic form $\omega_{0}$ passes to the resulting quotient space $T^{*} M / \Gamma$, since $\omega_{0}$ is invariant under translation by a closed 1 -form on $M$ and endows $T^{*} M / \Gamma$ with the structure of a regular almost cotangent structure.

The following results are the cotangent versions of Corollaries 3.4 and 3.5, and of Theorem 3.6, respectively.

Corollary 4.2. Let $(N, \Pi, M, \omega)$ be a regular almost cotangent structure with model fibre $T^{\rho} \times \mathbb{R}^{m-\rho}$. Suppose further that $N$ admits a global section $s$ of $N$ over $M$. Then $N$ is diffeomorphic with $T^{*} M / \Gamma$, where $\Gamma$ is a group isomorphic to $\mathbb{Z}^{\rho}$ arising from a collection of $\rho$ linearly independent closed 1-forms on $M$ in the manner described above. Moreover, under such an isomorphism $\omega$ is carried to $\omega_{0}+\phi$, where $\omega_{0}$ is the 2-form on $T^{*} M / \Gamma$ induced from $\omega_{0}$ on $T^{*} M$ and $\phi$ is the pullback from $M$ of a closed 2-form to $T^{*} M / \Gamma$.

Corollary 4.3. Let $(N, \Pi, M, \omega)$ be a regular almost cotangent structure.

(i) If the model fibre is compact and a smooth section of $N$ over $M$ exists, then $M$ is parallelizable.

(ii) If $N$ is compact and a smooth section of $N$ over $M$ exists, then $M$ is parallelizable.

Theorem 4.4. Let $(N, \Pi, M, \omega)$ be a regular integrable almost cotangent structure with fibre $F$. Then $\pi_{k}(N)$ and $\pi_{k}(M)$ are isomorphic for $k \geq 3$, and $\pi_{2}(N)$ is a subgroup of $\pi_{2}(M)$. Moreover, if $\pi_{1}(N)$ is trivial, $\pi_{1}(F)$ is isomorphic with $\pi_{1}(M) / \pi_{2}(N)$. In that case, if either $\pi_{2}(M)$ is trivial or $\pi_{2}(N)$ and $\pi_{2}(M)$ are isomorphic, then $(N, \Pi, M, \omega)$ is equivalent as an almost cotangent manifold to $T^{*} M$ and under such an equivalence $\phi: N \rightarrow T^{*} M,\left(\phi^{-1}\right)^{*} \omega$ differs from the canonical 2-form on $T^{*} M$ by the pullback of a closed 2-form on $M$.

Having noted that there are cotangent analogues of Theorem 3.3 and 3.6, it is natural to ask whether there is also an analogue of Theorem 3.9. Before discussing this, we prove the following theorem and consider two examples.

Theorem 4.5. Suppose that $(N, \Pi, M, \omega)$ is a regular almost cotangent structure. Then $(N, \Pi, M, \omega)$ is equivalent to the almost cotangent structure on $T^{*} M$ iff $\omega$ is exact, $\omega=d \theta$, say, with $\theta$ semibasic.

Proof. The necessity is obvious, so we consider the sufficiency aspect of the 
theorem. Consider then the unique vector field $\Delta$ which satisfies

$$
i(\Delta) d \theta=\theta \text {. }
$$

Then if $\alpha$ is any 1 -form on $M$ we have,

$$
\left\langle\Delta, \Pi^{*} \alpha\right\rangle=d \theta\left(\alpha^{v}, \Delta\right)=-d \theta\left(\Delta, \alpha^{v}\right)=-\left\langle\alpha^{v}, \theta\right\rangle=0,
$$

the last equality following from the fact that $\theta$ is semibasic. Thus $\Delta$ is a vertical vector field.

Next, choose a Riemannian metric $g$ on $M$. Then in the obvious way, $g$ engenders a Riemannian metric $g_{x}$ on the fibre $\Pi^{-1}(x)$. Furthermore, the inner product $g_{x}\left(\alpha^{v}, \beta^{v}\right)$ is constant on $\Pi^{-1}(x)$. Also, starting from (4.3) and computing the Lie derivative of each side along $\Delta$, we obtain for each 1 -form $\alpha$ on $M$

$$
\left[\alpha^{v}, \Delta\right]=\alpha^{v} .
$$

Finally, by computing the Lie derivative of the constant $g_{x}\left(\alpha^{v}, \beta^{v}\right)$ on the fibre along $\Delta$, we obtain

$$
L_{\Delta} g_{x}=2 g_{x},
$$

and the result follows just as Theorem 3.3 did from (3.10).

We note that in Theorem 4.4 the implicit hypothesis of completeness in the fibres of $\Pi$ is essential. Take, for example, $N$ to be $T^{*} \mathbb{R}^{m}$ with its zero section deleted, $M$ to be $\mathbb{R}^{m}$, and $\Pi$ and $\omega$ to be the restriction of, respectively, the cotangent map from $T^{*} \mathbb{R}^{m}$ to $\mathbb{R}^{m}$ and the canonical 2-form on $T^{*} \mathbb{R}^{m}$. Then $(N, \Pi, M, \omega)$ satisfies all the hypotheses of Theorem 4.4 except for the affine completeness of the fibres of $\Pi$, but it is clearly not equivalent to the almost cotangent structure on a cotangent bundle.

A more significant example of an exact almost symplectic manifold not diffeomorphic to a cotangent bundle is obtained as follows. Consider $\mathbb{C} P^{1}$ to consist of the set of complex lines through the origin in $\mathbb{C}^{2}$ and denote the natural map from $\mathbb{C}^{2} /\{0\}$ to $\mathbb{C} P^{1}$ by $\Pi$. Let $(z, w)=(x+i y, u+i v)$ be standard coordinates on $\mathbb{C}^{2}$ so that the almost complex structure $J$ on $\mathbb{C}^{2}$, realized as a 1-1 tensor field, is

$$
J=\frac{\partial}{\partial y} \otimes d x-\frac{\partial}{\partial x} \otimes d y+\frac{\partial}{\partial u} \otimes d v-\frac{\partial}{\partial v} \otimes d u .
$$

Next consider the 1-form $\theta$ given by

$$
\theta=x d u-y d v
$$

and its derivative $\omega$ given by

$$
\omega=d x \wedge d u-d y \wedge d v .
$$


It is easy to check that $\omega$ restricted to any complex line in a tangent space of $\mathbb{C}^{2} /\{0\}$ is zero. Hence, since the fibres of $\Pi$ are, by definition, complex lines, $\omega$ vanishes on fibres of $\Pi$; in other words, $\omega$ is Lagrangian with respect to the fibres of $\Pi$.

We have shown above that $\left(\mathbb{C}^{2} /\{0\}, \Pi, \mathbb{C} P^{1}, \omega\right)$ is an exact integrable almost cotangent structure. Clearly the fibres of $\Pi$, though locally affine, are not affine-complete. Furthermore, $\omega$ does not extend to a Lagrangian 2-form on the fibrewise-completion of $\left(\mathbb{C}^{2} /\{0\}, \Pi, \mathbb{C} P^{1}, \omega\right)$. For on the one hand, the fibres of the completed bundle would be diffeomorphic to $\mathbb{R}^{2}$ and so the completion would, by Theorem 4.1 , be $T^{*} \mathbb{C} P^{1}$. And on the other hand, the completion would by construction be the canonical complex line bundle $\gamma_{1}^{1}$ over $\mathbb{C} P^{1}$, which is known to be inequivalent as a (vector) bundle to $T^{*} \mathbb{C} P^{1}$.

Prior to Theorem 4.5, we asked if there were some cotangent analogue of Theorem 3.3. Theorem 4.5 is to some extent such an analogue. The analogy would, however, be even closer if one did not have to make the assumption that the 1 -form $\theta$ is semibasic. Unfortunately, it seems to be necessary to make this assumption at least in the proof given here. (One could try, for example, to argue that $\theta$ could always be modified so as to be semibasic; however, it is not hard to show that the obstruction to making such a modification lies in $H^{2}(M, \mathbb{Z})$. If the latter group were to vanish, then of course Theorem 4.4 would be applicable. In the case where $\pi_{1}(M)$ were trivial, using also the Hurewicz and universal coefficient theorems, we could conclude that $\pi_{2}(M)$ was also trivial and so $N$ would be covered by a cotangent bundle).

Theorems 4.1, 4.4, and 4.5 suggest the following question: do there exist regular almost cotangent manifolds $(N, \pi, M, \omega)$ such that, to use notation similar to that used prior to Theorem $3.6, N$ is not covered by $T^{*} M$ ? Without being able to answer this question in full generality, we shall show that if there exists such an example then $m \geq 3$. First, we can exclude the case $m=1$, for the only possibilities for $N$ are $\mathbb{R}^{2}$, which is $T^{*} \mathbb{R}$, and $S^{2}$, which is ruled out by the remarks made at the end of $\S 2$. Second, for $m=2$ we must have $M$ diffeomorphic to $\mathbb{R}^{2}$ or $S^{2}$. In the first case $\pi_{2}(M)$ is trivial and we can apply Theorem 4.4. On the other hand, if $M$ is diffeomorphic to $S^{2}$ we have a further three cases to consider, namely, according as the fibre $F$ of $\Pi$ is diffeomorphic to $\mathbb{R}^{2}, T^{2}$ or $\mathbb{R} \times S^{1}$. In the first case, $N$ must be diffeomorphic to $T^{*} S^{2}$ by Theorem 4.1 ( $N$ admits a section over $M$ since $\mathbb{R}^{2}$ is contractible). Moreover, $F$ cannot be diffeomorphic to $T^{2}$, because we know that $\pi_{1}(F)=\pi_{2}(M) / \pi_{2}(N)$, which is inconsistent with the fact that $\pi_{1}\left(T^{2}\right)=\mathbb{Z} \times \mathbb{Z}$ and $\pi_{2}\left(S^{2}\right)=\mathbb{Z}$.

There remains the possibility that $F$ is diffeomorphic to $\mathbb{R} \times S^{1}$. In that case, for $x \in S, \Pi^{-1}(x)$ is a one-dimensional lattice in $T_{x}^{*} S^{2}$. The elements in $T_{x} S^{2}$ annihilated by each element in this lattice form a one-dimensional subspace and taking the union over each $x \in S^{2}$ gives a rank one subbundle of $T S^{2}$, a condition which is well known to be impossible [2, 21]. 


\section{ACKNOWLEDGMENTS}

We would like to thank Professors E. G. Rees, M. Crampin, and R. Howard for useful conversations and the referee for some very constructive suggestions concerning this work. Both authors gratefully acknowledge the support of the Science and Engineering Research Council of Great Britain, and one of us (G.T.) thanks the mathematics department of the University of North Carolina at Chapel Hill for its hospitality during the completion of this work.

\section{REFERENCES}

1. J. Beem, Proper homothetic maps and fixed points, Lett. Math. Phys. 2 (1978), 317-320.

2. R. Bott and T. Loring, Differential forms in algebraic topology, Springer, 1980.

3. F. Brickell and R. S. Clark, Integrable almost tangent structures, J. Differential Geom. 9 (1974), 557-563.

4. M. R. Bruckheimer, Doctoral thesis, University of Southampton, 1960.

5. R. S. Clark and D. S. Goel, On the geometry of an almost tangent manifold, Tensor (N.S.) 24 (1972), 243-252.

6. __ Almost cotangent manifolds, J. Differential Geom. 9 (1974), 109-122.

7. M. Crampin, Tangent bundle geometry for Lagrangian dynamics, J. Phys. A 16 (1983), 3755-3772.

8. M. Crampin and G. Thompson, Affine bundles and integrable almost tangent structures, Math. Proc. Cambridge Philos. Soc. 98 (1985), 61-71.

9. M. Crampin and L. A. Ibort, Graded Lie algebras of derivations and Ehresmann connections, J. Math. Pures Appl. 66 (1987), 113-125.

10. H. A. Eliopuolos, Structures presque tangente sur les variétés différentiables, C. R. Acad. Sci. Paris 255 (1962), 1563-1565.

11. _ On the general theory of differentiable manifolds with almost tangent structure, Canad. Math. Bull. 8 (1965), 721-748.

12. J. Grifone, Structure presque-tangente et connexions. I, Ann. Inst. Fourier (Grenoble) 22 (1972), 287-334.

13. __ Structure presque-tangente et connexions. II, Ann. Inst. Fourier (Grenoble) 22 (1972), 291-388.

14. V. Guillemin and S. Sternberg, Geometric asymptotics, Math. Surveys, vol. 14, Amer. Math. Soc., Providence, R.I., 1977.

15. __ Symplectic techniques in physics, Cambridge Univ. Press, 1984.

16. J. Klein, Espaces variationels et mécanique, Ann. Inst. Fourier (Grenoble) 12 (1962), 1-124.

17. __ Structures symplectiques ou J-symplectiques homogènes sur l'espace tangent, Symposia Math. 14 (1974), 181-192.

18. _ Geometry of sprays. Lagrangian case principle of least curvature, Proc. IUTAM-ISIMM Symposium on Modern Developments in Analytical Mechanics, Torino, 1982.

19. S. Kobayashi, Transformation groups in differential geometry, Springer, 1972.

20. D. Laugwitz, Differential and Riemannian geometry, Academic Press, New York, 1965, pp. 159.

21. J. Milnor and J. D. Stasheff, Characteristic classes, Princeton Univ. Press, Princeton, N. J., 1972.

22. S. Sternberg, Lectures in differential geometry, Prentice-Hall, Englewood Cliffs, N. J., 1964.

23. G. Thompson, Integrable almost cotangent structures and Legendrian bundles, Math. Proc. Cambridge Philos. Soc. 101 (1987), 61-78. 
24. A. Weinstein, Lectures on symplectic manifolds, CBMS Regional Conf. Ser. in Math., no. 29, Amer. Math. Soc., Providence, R. I., 1977.

25. N. M. J. Woodhouse, Geometric quantization, Oxford Univ. Press, Oxford, 1980.

Department of Mathematics, University of Edinburgh, Edinburgh EH9 3JZ, Great BRITAIN

Current address, G. Thompson: Department of Mathematics, University of Toledo, Toledo, Ohio 43606 